\title{
The dynamics of two-circle and three-circle inversion
}

\author{
by
}

\author{
Daniel M. Look (Indiana, PA)
}

\begin{abstract}
We study the dynamics of a map generated via geometric circle inversion. In particular, we define multiple circle inversion and investigate the dynamics of such maps and their corresponding Julia sets.
\end{abstract}

0. Introduction. The goal of this work is to extend the notion of geometric circle inversion to multiple circles and examine the dynamics of the resulting maps under iteration. We will use the notation $f^{n}(z)$ for $f \circ \cdots \circ f(z)$ where the composition occurs $n$ times. We are particularly interested in the structure of the Fatou and Julia sets of the circle inversion maps. The Julia set for a rational map $R(z)$, denoted $J(R)$, is the set of points where the iterates of $R(z)$ do not form a normal family in the sense of Montel (see [3] for more details). For rational maps of degree greater than or equal to 2 the Julia set coincides with the closure of the set of repelling cycles of $R(z)$. The Fatou set is the complement of the Julia set, so $\mathbb{C}_{\infty}-J(R)$ where $\mathbb{C}_{\infty}=\mathbb{C} \cup\{\infty\}$. The Julia set is where the iterates behave chaotically, while the Fatou set consists of points where the function iterates behave calmly.

We will begin with the standard definition of geometric circle inversion. Here the inversion of a point $\alpha$ about a circle centered at $O$ with radius $r$ is the point $\alpha^{\prime}$ on the ray $\overrightarrow{O \alpha}$ such that

$$
\frac{\|\overrightarrow{O \alpha}\|}{r}=\frac{r}{\left\|\overrightarrow{O \alpha^{\prime}}\right\|},
$$

where $\|\overrightarrow{O \alpha}\|$ is the distance between $O$ and $\alpha$. Let us say that we have $n$ circles, which we will refer to as the generating circles, $C_{1}, \ldots, C_{n}$. We will define $n$-circle inversion as sending the point $\alpha$ to the point $\beta$ that is the

2000 Mathematics Subject Classification: Primary 37F10; Secondary 51N05, 37F45.

Key words and phrases: Julia set, complex dynamics, geometry, circle inversion, topology. 
arithmetic mean of $\alpha_{1}, \ldots, \alpha_{n}$ where $\alpha_{i}$ is the inversion of $\alpha$ about circle $C_{i}$ (see Figure 1).

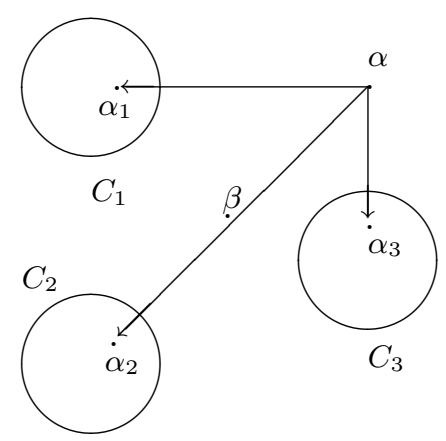

Fig. 1. $\beta$ is the inversion of $\alpha$ about the three circles $C_{1}, C_{2}, C_{3}$

In this work we will examine the special case that arises when the circles are centered on the $n$th roots of unity and all have the same radius $r$. This is in an effort to decrease the number of parameters; the case with differing radii will be investigated in a later work. In particular, we will work with $n=2,3$. When we examine the dynamics of these maps we will see that, like the classic quadratic case $Q_{c}(z)=z^{2}+c$, there are only two forms of hyperbolic Julia sets possible: Cantor sets or connected sets. Unlike the Julia sets for $Q_{c}$ the dynamics on the connected Julia sets arising from $n$-circle inversion (for a given $n=2,3$ ) are all conjugate. Another important difference is that our map's only parameter, the common radius $r$, will be real and positive.

When $n=2$ the circle inversion map has a Cantor set Julia set when $r<1$. As the radius grows through the value 1 there is a geometric bifurcation that occurs as our generating circles become tangent and then intersect. Simultaneously there is a functional bifurcation that occurs as the map undergoes a saddle-node bifurcation. As this occurs the Julia set closes and becomes the entire extended real axis. When $n=3$ we see a slightly different occurrence. The Julia set for this map is also a Cantor set when $r$ is small, in this case when $r<r_{0}=3^{1 / 2} 2^{-1 / 3} \approx 1.37473$. A functional bifurcation occurs as $r$ passes through $r_{0}$ with the map undergoing a saddle-node bifurcation in which the Julia set changes from a Cantor set to a connected set. Unlike the $n=2$ case the functional bifurcation does not coincide with the geometric bifurcation occurring when the circles become tangent $(r=\sqrt{3} / 2 \approx 0.866)$.

I would like to thank the referee for providing me with a number of extremely helpful comments on the original version of this paper. 


\section{Two circles}

1.1. Preliminaries for two circles. We will examine the case of two generating circles, each with radius $r$, centered at \pm 1 . Our map is then

$$
z \mapsto \frac{r^{2} \bar{z}}{\bar{z}^{2}-1} .
$$

Since we want to have access to the tools of holomorphic dynamics we will define our map to be

$$
F_{r}(z)=\frac{r^{2} z}{z^{2}-1} .
$$

This map agrees on every second iterate with, and therefore will have the same Julia and Fatou sets as, the "true" circle inversion map (Theorem 4.2 of [3]). (The dynamical similarities between $F_{r}$ and the true circle inversion map resemble the similarities between $z \mapsto z^{2}$ and $z \mapsto 1 / z^{2}$.) The graph of $F_{r}(z)$ restricted to the real axis (along with the identity $I(x)=x$ ) is shown in Figure 2.

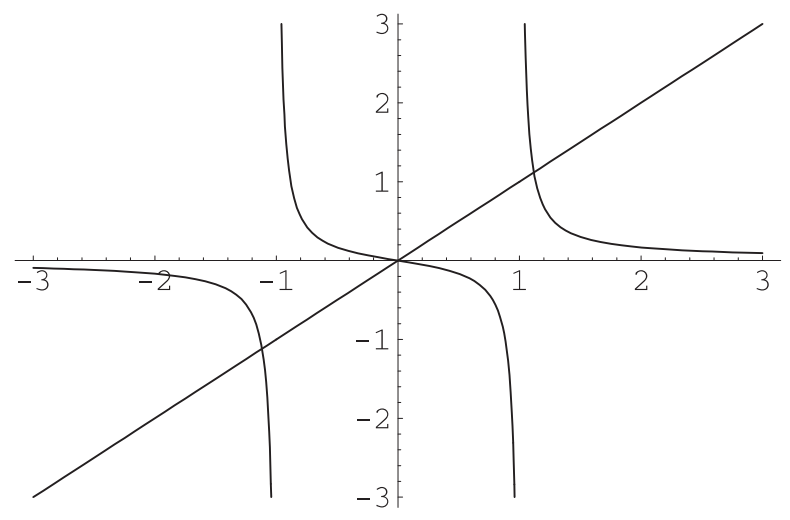

Fig. 2. $F_{r}$ with $r=0.5$

For any $r$ we have poles at \pm 1 , the centers of the generating circles, and fixed points at 0 and $\pm \sqrt{1+r^{2}}$. Let $p=\sqrt{1+r^{2}}$. An easy calculation shows that the fixed point at 0 is attracting if $0<r<1$ and repelling if $r>1$ while the fixed points $\pm p$ are always repelling. It is worth noting that a topological bifurcation occurs at $r=1$ as the generating circles are disjoint if $r<1$, tangent if $r=1$ and intersecting if $r>1$. The critical points of $F_{r}$ are always $\pm i$. Since $-F_{r}(i)=F_{r}(-i)$ we have, essentially, only one free critical orbit. Throughout this work we will denote the set $\mathbb{R} \cup\{\infty\}$ by $\mathbb{R}_{\infty}$.

Theorem 1.1. $J\left(F_{r}\right) \subseteq \mathbb{R}_{\infty}$ for all $r>0$.

Proof. For all $r>0$ the set $\mathbb{R}_{\infty}$ is forward and backward invariant under $F_{r}$. Further, $\pm p \in \mathbb{R}_{\infty}$ are repelling fixed points and are therefore 
in $J\left(F_{r}\right)$. The iterated pre-images of any point in the Julia set are dense in the Julia set (Theorem 4.10 of [3]), implying that $J\left(F_{r}\right) \subseteq \mathbb{R}_{\infty}$ since the iterated pre-images of $\pm p$ are contained in $\mathbb{R}_{\infty}$.

To avoid confusion we will refer to the map as $F_{r}(x)$ when we wish to consider our map on the real axis only.

1.2. Two non-intersecting generating circles. In this section we will focus on the case $0<r<1$. Recall that for these $r$-values the fixed point at the origin is attracting.

TheOREM 1.2. For $0<r<1$ the imaginary axis, $i \mathbb{R}$, is contained in the immediate basin of attraction for 0 .

Proof. Since

$$
F_{r}(i z)=\frac{r^{2} z}{-1-z^{2}} i
$$

the imaginary axis is invariant under $F_{r}$ and, on the imaginary axis, our map is

$$
y \mapsto \frac{-r^{2} y}{1+y^{2}}
$$

where $y \in \mathbb{R}$. A simple calculation shows that whenever $y^{2}>r^{2}-1$ we have

$$
\left|\frac{-r^{2} y}{1+y^{2}}\right|<|y|
$$

implying that all points on the imaginary axis are sent closer to the origin. Since we are looking at the case $r<1$ we know that for all real $y, y^{2}$ will be greater than $r^{2}-1$. Hence, the entire imaginary axis is in the immediate basin of attraction for 0 .

The critical points for this map are $\pm i$ and therefore we have:

Corollary 1.3. For $0<r<1$, all of the critical points for the map $F_{r}$ are in the immediate basin of attraction of 0 .

Therefore $F_{r}$ is a degree two map whose critical points are all in the immediate basin of attraction of one attracting fixed point, implying that $J\left(F_{r}\right)$ is a Cantor set (see Theorem 9.8.1 in [1]).

We can also use symbolic dynamics to obtain this result and gain further insight into the dynamics of $F_{r}$ on $J\left(F_{r}\right)$. For the remainder of this section, we will restrict our attention to the real axis.

Lemma 1.4. For $0<r<1$ there exist disjoint intervals $A_{0}$ and $A_{1}$ in $\mathbb{R}$ such that the following holds: each $A_{i}$ maps in a one-to-one fashion over both $A_{0}$ and $A_{1}$ and the Julia set of $F_{r}$ is contained in $A_{0} \cup A_{1}$.

Proof. Since 0 is an attracting fixed point for $F_{r}(x)$ for all $0<r<1$ we know that there exists a point $\omega \in(0,1)$ such that $\omega$ is in the immediate 
basin of attraction for 0 . Using the fact that $F_{r}(x)$ is odd we see that the interval $[-\omega, \omega]$ is in the immediate basin of attraction for 0 . Let $\Lambda$ denote the complement of $[-\omega, \omega]$ in $\mathbb{R}_{\infty}$.

The interval $(0,1)$ is mapped in one-to-one fashion over the interval $(-\infty, 0)$, implying that there exists a pre-image of $-\omega$ in $(0,1)$. Call this pre-image $\alpha_{-}$. Further, $(1, \infty)$ is mapped in one-to-one fashion over the interval $(0, \infty)$, implying that there exists a pre-image of $\omega$ in $(1, \infty)$. We will call this pre-image $\alpha_{+}$. Let $A_{1}=\left(\alpha_{-}, \alpha_{+}\right)$and $A_{0}=-A_{1}$ (see Figure 3). Note that neither $\alpha_{-}$nor $\alpha_{+}$are in $[-\omega, \omega]$ since all points in $[-\omega, \omega]$ move closer to 0 upon iteration while $\alpha_{-}$is mapped to $-\omega$ and $\alpha_{+}$is mapped to $\omega$. Since neither $\alpha_{-}$nor $\alpha_{+}$are in $[-\omega, \omega]$ and the interval $A_{1}$ does not contain 0 we know that $A_{1} \cap[-\omega, \omega]=\emptyset$, implying that $A_{1} \subset \Lambda$. Hence, since $-\Lambda=\Lambda, A_{i} \subset \Lambda$ for $i=0,1$. By construction the endpoints of $A_{i}$ (for $i=0,1$ ) are mapped to the endpoints of $[-\omega, \omega]$ and each $A_{i}$ contains a pole, implying that the interval $A_{i}$ is mapped over $\Lambda$ for $i=0,1$. Since $F_{r}^{\prime}(x)$ is bounded away from 0 on $A_{1}$ we know that this mapping is one-to-one.

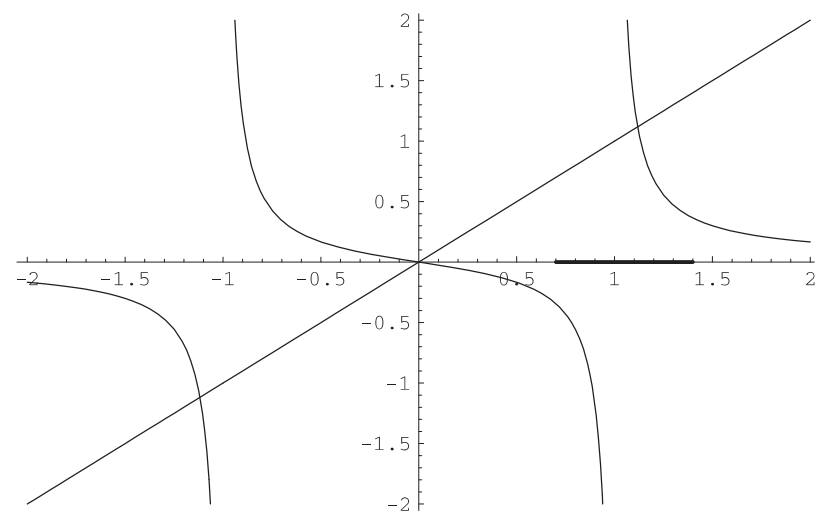

Fig. 3. The region $A_{1}$ (shaded)

The complement of $F_{r}\left(A_{i}\right)=\Lambda$ for $i=1,2$ is the interval $[-\omega, \omega]$, which is contained in the immediate basin of 0 . Hence $J\left(F_{r}\right) \subset A_{0} \cup A_{1}$ and $J\left(F_{r}\right)$ is precisely the set of points whose orbits remain in $A_{0} \cup A_{1}$ for all time (and are hence not attracted to 0 ).

TheOREM 1.5. For $0<r<1$ the Julia set of $F_{r}(x)$ is a Cantor set and $F_{r} \mid J\left(F_{r}\right)$ is conjugate to the one-sided shift on two symbols.

Proof. To each point $z \in J\left(F_{r}\right)$ we can assign a sequence $s(z)=s_{1} s_{2} s_{3} \ldots$ using the rule $F_{r}^{i}(z) \in A_{s_{i}}$. We need to show that each sequence corresponds to a point in the Julia set and that no sequence corresponds to multiple 
points. Since $F_{r}$ maps each $A_{i}$ over both of the $A_{i}$ 's we know that each sequence will correspond to at least one point in the Julia set.

Since the critical points are bounded away from the real line (and hence from the Julia set) we know that $F_{r}$ is dynamically hyperbolic on its Julia set. This expansion guarantees that no sequence can correspond to multiple points.

The map that takes the sequence $s_{0} s_{1} s_{2} \ldots$ to the corresponding point in $J\left(F_{r}\right)$ defines a homeomorphism between the space of one-sided sequences of 0's and 1's and the Julia set, $J\left(F_{r}\right)$. Hence, $J\left(F_{r}\right)$ is a Cantor set and standard arguments (see [2], for example) show that $F_{r} \mid J\left(F_{r}\right)$ is conjugate to the one-sided shift on two symbols.

We have therefore shown:

THEOREM 1.6. The Julia set for the two-circle inversion map for nonintersecting circles is a Cantor set on the line through the centers of both circles on which the map is conjugate to a one-sided shift on two symbols.

1.3. Two intersecting generating circles. When $r>1$ our generating circles intersect. The topological bifurcation that occurs as $r$ increases through 1 and our circles begin to intersect corresponds to a dynamical bifurcation as the fixed point at the origin ceases to be attracting. There is a two-cycle given by $\pm \sqrt{1-r^{2}}$. When $r<1$, this cycle is real and repelling. The twocycle coalesces with the origin when $r=1$ and emerges on the imaginary axis when $r>1$. Note that

$$
\left|F_{r}^{\prime}\left(\sqrt{1-r^{2}}\right) F_{r}^{\prime}\left(-\sqrt{1-r^{2}}\right)\right|=\left|\frac{\left(r^{2}-2\right)^{2}}{r^{4}}\right|=\frac{\left(r^{2}-2\right)^{2}}{r^{4}} .
$$

When $r>1,-4 r^{2}+4<0$, implying that $r^{4}-4 r^{2}+4<r^{4}$. Therefore,

$$
\frac{\left(r^{2}-2\right)^{2}}{r^{4}}=\frac{r^{4}-4 r^{2}+4}{r^{4}}<1
$$

and hence the two-cycle given by $\pm \sqrt{1-r^{2}}$ is attracting for all $r>1$.

TheOREM 1.7. For all $r>1$, both critical points are attracted to the two-cycle $\pm \sqrt{1-r^{2}}$ and all components of the Fatou set are pre-images of the basin of attraction for this two-cycle.

Proof. Since the attracting cycle $\pm \sqrt{1-r^{2}}$ must attract a critical point we know that one of the critical points must be in the basin of attraction for this two-cycle. The critical points are $\pm i$ and $F_{r}(-z)=-F_{r}(z)$. Hence, one of the critical points being in the basin of attraction for $\pm \sqrt{1-r^{2}}$ implies both are in that basin. Therefore, both of our critical points are attracted to the two-cycle $\pm \sqrt{1-r^{2}}$. Hence, the Fatou set for this map is the union of all of the backwards images of the immediate basin of attraction for the two-cycle $\pm \sqrt{1-r^{2}}$. 
Theorem 1.8. $J\left(F_{r}\right)=\mathbb{R}_{\infty}$ for $r>1$.

Proof. By Theorem 1.1 we know that $J\left(F_{r}\right) \subseteq \mathbb{R}_{\infty}$. It will suffice to show that the intersection of the Fatou set and $\mathbb{R}_{\infty}$ is empty. Since the Fatou set consists only of the pre-images of the immediate basin of attraction for the attracting two-cycle on the imaginary axis and $\mathbb{R}_{\infty}$ is fully invariant we know that no points of $\mathbb{R}_{\infty}$ are attracted to this two-cycle. Hence, the intersection of the Fatou set and $\mathbb{R}_{\infty}$ is empty.

This implies that the Fatou set for $F_{r}$ has only two components: the upper half-plane $\{z: \operatorname{Im}(z)>0\}$ and the lower half-plane $\{z: \operatorname{Im}(z)<0\}$, which correspond to the two components in the basin of attraction for the two-cycle $\pm \sqrt{1-r^{2}}$.

Since $J\left(F_{r}\right)=\mathbb{R}_{\infty}$ we will restrict our attention to $F_{r}(x)=F_{r}(z) \mid \mathbb{R}$. Let $I_{0}=\mathbb{R}^{-} \cup\{0\} \cup\{\infty\}$ and $I_{1}=\mathbb{R}^{+} \cup\{0\} \cup\{\infty\}$. The map $F_{r}$ takes $I_{i}(i=0,1)$ in one-to-one fashion onto $\mathbb{R}_{\infty}$. To each point $x \in J\left(F_{r}\right)$ we can assign a sequence $s(x)=s_{1} s_{2} s_{3} \ldots$ using the rule $F_{r}^{i}(x) \in I_{s_{i}}$. Since $I_{0} \cap I_{1}=\{0, \infty\}$ the points 0 and $\infty$ (along with their pre-images) will be associated with multiple sequences. Hence, we will be able to show that $F_{r} \mid J\left(F_{r}\right)$ is conjugate to a quotient of a one-sided shift on two symbols.

THEOREM 1.9. When $r>1$ there is a conjugacy between $F_{r}(z) \mid J\left(F_{r}\right)$ and a quotient of the one-sided shift map on two symbols.

Proof. We need to show that each sequence corresponds to a point in the Julia set and that no sequence corresponds to multiple points. Since $F_{r}$ maps each $I_{i}$ over both of the $I_{i}$ 's we know that each sequence will correspond to at least one point in the Julia set.

Since the Julia set is $\mathbb{R}_{\infty}$ and the critical points are bounded away from $\mathbb{R}$ we know that $F_{r}$ is dynamically hyperbolic on its Julia set. This expansion guarantees that no sequence can correspond to multiple points.

Note that the sequences $\overline{10}$ and $\overline{01}$ both correspond to 0 . The pre-image of 0 is $\infty$, which is represented by the sequences $0 \overline{01}$ and $1 \overline{10}$. Finally, any point whose orbit includes the poles \pm 1 will also have two sequences associated with it: $\alpha 0 \overline{01}$ and $\alpha 1 \overline{10}$. If we identify these points in shift space we have a conjugacy between this quotient of the one-sided shift on two symbols and our map restricted to its Julia set.

We have therefore shown:

THEOREM 1.10. The Julia set for the two-circle inversion map for intersecting circles is the entire line through the centers of both circles on which the map is conjugate to a quotient of the one-sided shift on two symbols.

The symbolic dynamics for $F_{r} \mid J\left(F_{r}\right)$ for $r>1$ are identical to those arising on the Julia set for the map $h(z)=1 / z^{2}$. The Julia set for $h(z)$ 
is the unit circle on which we can place symbolic dynamics by letting $I_{0}$ be the closed upper semicircle while $I_{1}$ is the closed lower semicircle. The symbolic dynamics generated are identical to those of $F_{r}$ on its Julia set when $r>1$. Under $h(z)$ the point 1 , being in $I_{0} \cap I_{1}$, is associated with two sequences: $\overline{01}$ and $\overline{10}$. The point -1 is also in $I_{0} \cap I_{1}$ and is represented by the sequences $0 \overline{01}$ and $1 \overline{10}$. Further, just as there are only two components of the Fatou set for $F_{r}$ with $r>1$, there are exactly two components of the Fatou set for $h(z)$ : the interior and exterior of the unit circle, which are the two components of the basin of attraction for the two-cycle $\{0, \infty\}$.

\section{Three generating circles}

2.1. Construction of the map. We now shift our attention to the case of three generating circles. As in the case of two generating circles, we will assume that all three generating circles have the same radius $r$ and are positioned symmetrically. Without loss of generality, assume that the generating circles are each centered at a cube root of unity. Our map is then

$$
z \mapsto \frac{r^{2} \bar{z}^{2}}{\bar{z}^{3}-1} .
$$

As in the preceding section we wish our map to be holomorphic. Hence, we will define our map as

$$
G_{r}(z)=\frac{r^{2} z^{2}}{z^{3}-1}
$$

As in the two-circle case, this map agrees on every second iterate with, and therefore will have the same Julia and Fatou sets as, the "true" circle inversion map (Theorem 4.2 of [3]).

2.2. Preliminaries for three generating circles. Throughout the remainder of this work we will consider $\omega$ such that $\omega^{3}=1$ and $\omega \neq 1$. The map $G_{r}$ has 3 -fold symmetry in the sense that $G_{r}(\omega z)=\omega^{2} G_{r}(z)$. Hence, we know that if $z_{0}$ is attracted to a periodic cycle, then $\omega z_{0}$ and $\omega^{2} z_{0}$ are also attracted to periodic cycles, although they could be different cycles (and even of different periods). Further, $\mathbb{R}_{\infty}$ is forward invariant under $G_{r}$ and $G_{r}$ maps the line $\omega \mathbb{R}_{\infty}$ to $\omega^{2} \mathbb{R}_{\infty}$ and vice versa. Hence, $\omega \mathbb{R}_{\infty} \cup \omega^{2} \mathbb{R}_{\infty}$ is also a forward invariant set. We will call $\omega \mathbb{R}_{\infty}$ and $\omega^{2} \mathbb{R}_{\infty}$ the symmetric axes. As a final symmetry, note that $\overline{G_{r}(z)}=G_{r}(\bar{z})$.

The family $G_{r}$ always has a superattracting fixed point at the origin. Since $G_{r}$ is of degree three there are four critical points. One of these critical points is always in the negative real axis; we will denote this critical point by $c$. The four critical points are then $0, c, \omega c$ and $\omega^{2} c$. Since 0 is fixed, we will call the three symmetric critical points $c, \omega c$, and $\omega^{2} c$ the free critical points. By our symmetries we know that $G_{r}(\omega c)=\omega^{2} G_{r}(c)$ and $G_{r}\left(\omega^{2} c\right)=\omega G_{r}(c)$. 
This implies that $G_{r}$ has (essentially) only one free critical orbit in the sense that if one of the free critical points is in the basin of attraction for 0 then all of them are. Similarly, if one of the free critical points has bounded orbit then so do all of them. Further, for all values of $r, G_{r}$ has poles at the cube roots of unity.

2.3. Structure of $J\left(G_{r}\right)$ for $r$ small. Let $r_{0}=3^{1 / 2} 2^{-1 / 3} \approx 1.37473$. The map $G_{r}$ undergoes a saddle-node bifurcation as $r$ passes through $r_{0}$. Figure 4 shows the graph of $G_{r}$ restricted to the real axis for $r<r_{0}$ and $r>r_{0}$.
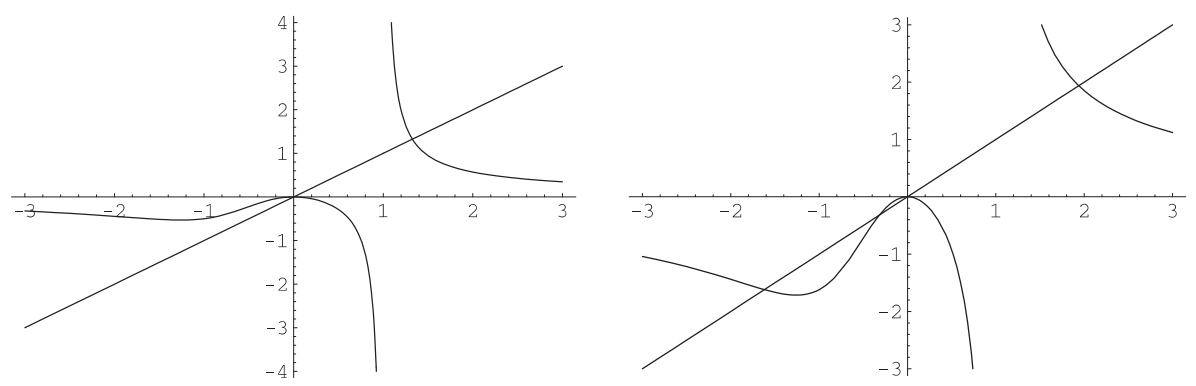

Fig. 4. The bifurcation as $r$ passes through $r_{0}$

TheOREM 2.1. For $r<r_{0}$ the negative real axis is in the immediate basin of attraction for 0 .

Proof. We will prove this by showing that for $r<r_{0}$ we have $\left|G_{r}(x)\right|$ $<|x|$ for all $x \in \mathbb{R}^{-}$. The minimum value of $x^{2}-1 / x$ on $\mathbb{R}^{-}$is $3 \cdot 2^{-2 / 3}$. Since $r_{0}=3^{1 / 2} 2^{-1 / 3}, r<r_{0}$ implies that $r^{2}<3 \cdot 2^{-2 / 3} \leq x^{2}-1 / x$ for $x \in \mathbb{R}^{-}$. Hence, for $x \in \mathbb{R}^{-}$and $r<r_{0}$, we have $r^{2}<x^{2}-1 / x$, implying that $r^{2} x^{2}<x^{4}-x=x\left(x^{3}-1\right)$. Since $x \in \mathbb{R}^{-}$we know that $x^{3}-1<0$, implying

$$
\frac{r^{2} x^{2}}{x^{3}-1}>x
$$

This yields

$$
\left|\frac{r^{2} x^{2}}{x^{3}-1}\right|<|x|
$$

since $G_{r}(x)<0$ when $x<0$. Ergo $\left|G_{r}(x)\right|<|x|$ for $x \in \mathbb{R}^{-}$and $r<r_{0}$, implying that $\mathbb{R}^{-}$is in the immediate basin of attraction for 0 .

Since $c \in \mathbb{R}^{-}$we know $c$ is in the immediate basin of attraction for 0 . Our 3-fold symmetry guarantees that $\omega c$ and $\omega^{2} c$ are also in the immediate basin of 0 . Therefore $G_{r}$ is a degree three map whose critical points are all attracted to a superattracting fixed point, implying that $J\left(G_{r}\right)$ is a Cantor set (see Theorem 9.8.1 in [1]). As before, we can also use symbolic dynamics to achieve this result and obtain further insight into the map's behavior. 
Let $D$ be a simply connected open set containing the origin such that $D$ is mapped two-to-one into itself. We know that such a set exists since 0 is a superattracting fixed point of order 2. Further, we will choose $D$ such that the boundary of $D$, which we will denote $\delta$, is a simple closed curve containing no critical points or critical values. Now, $G_{r}(\delta)$ is a simple closed curve contained within $D$ and bounding $G_{r}(D)$. We note that $G_{r}(D)$ has two pre-images, $D$ (mapped two-to-one over $G_{r}(D)$ ) and another simply connected (on $\mathbb{C}_{\infty}$ ) open set (mapped one-to-one over $G_{r}(D)$ ) that contains $\infty$. We will denote this second pre-image by $D_{\infty}$. Let $\delta_{\infty}$ denote the boundary of $D_{\infty}$. Hence, $G_{r}(D)=G_{r}\left(D_{\infty}\right)$ and this set is in the basin of attraction for 0 . Therefore, the Julia set for $G_{r}$ must be contained within the closed annular region between $\delta$ and $\delta_{\infty}$. We will denote this region by $\mathcal{A}$. Note that, by continuity, we know that the inner and outer boundaries of $\mathcal{A}$ are both mapped onto $G_{r}(\delta) \subset D$. The minimum modulus principle can be used to show that $G_{r}(\mathcal{A})=\mathbb{C}_{\infty}-G_{r}(D)$.

Let $P_{1}$ be the closure of the set of points $z \in \mathcal{A}$ such that $-\pi / 3 \leq$ $\arg z \leq \pi / 3$ (see Figure 5). Let $\varrho=\partial P_{1} \cap \omega^{2} \mathbb{R}^{-}, \gamma=\partial P_{1} \cap \delta$, and $\gamma_{\infty}=\partial P_{1} \cap \delta_{\infty}$. Notice that the boundary of $P_{1}$ is given by $\varrho \cup \bar{\varrho} \cup \gamma \cup \gamma_{\infty}$ (see Figure 5).

Note that $\gamma$ is mapped onto the points of $\partial G_{r}(\delta)$ with argument between $\pi / 3$ and $5 \pi / 3$ while $\gamma_{\infty}$ is mapped onto the points of $\partial G_{r}(\delta)$ with argument between $-\pi / 3$ and $\pi / 3$.

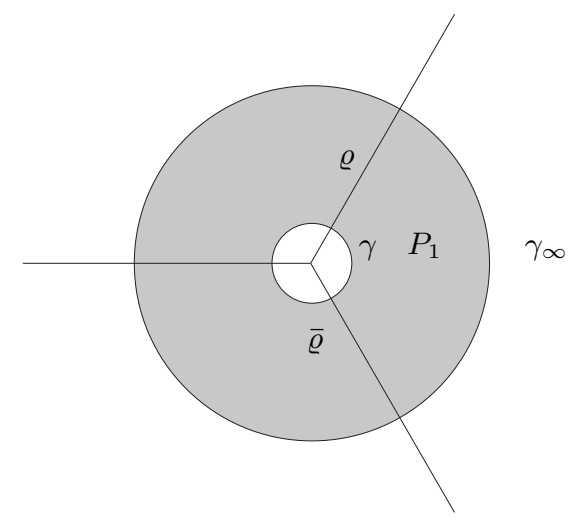

Fig. 5. The region $P_{1}$ and its boundary components

So $\gamma \cup \gamma_{\infty}$ is mapped over $\partial G_{r}(\delta)$ in one-to-one fashion except that $G_{r}(\gamma \cap \varrho)=G_{r}\left(\gamma_{\infty} \cap \varrho\right)$ and $G_{r}(\gamma \cap \bar{\varrho})=G_{r}\left(\gamma_{\infty} \cap \bar{\varrho}\right)$. Further, $\varrho$ is mapped two-to-one (except at the critical value) over the portion of $\bar{\varrho}$ between $\bar{\varrho} \cap \partial G_{r}(\delta)$ and the critical value on $\bar{\varrho}$. Hence, by symmetry, we know that $\bar{\varrho}$ is mapped two-to-one (except at the critical value) over the portion of $\varrho$ between $\varrho \cap \partial G_{r}(\delta)$ and the critical value on $\varrho$. 
By the maximum modulus principle we know that $\partial\left[G_{r}\left(P_{1}\right)\right]$ is given by $G_{r}\left(\partial P_{1}\right)$. There are no zeros in $P_{1}(0$ and $\infty$ are the only zeros $)$ so the interior of $P_{1}$ must be mapped over the entire unbounded complement of $G_{r}\left(\partial P_{1}\right)$. Hence, $P_{1}$ itself is mapped over $\mathbb{C}_{\infty}-G_{r}(D)$ in essentially one-to-one fashion (the map is one-to-one except along the rays $\varrho$ and $\varrho$ ). Therefore, all of $\mathcal{A}$ is contained in $G_{r}\left(P_{1}\right)$. By symmetry we can define $P_{2}=\omega P_{1}$ and $P_{3}=\omega P_{1}$ and it will be the case that $\mathcal{A}$ is contained in $G_{r}\left(P_{i}\right)$ for $i=1,2,3$.

Now recall that all of $\mathbb{R}^{-}$is in the immediate basin of attraction for 0 . By symmetry, the symmetric axes $\omega \mathbb{R}^{-}$and $\omega^{2} \mathbb{R}^{-}$are also in the immediate basin of 0 . Since the connected closed set $W=\{0\} \cup \mathbb{R}^{-} \cup \omega \mathbb{R}^{-} \cup \omega^{2} \mathbb{R}^{-}$is in the basin of attraction for 0 we can find an open connected set $U$ containing $W$ such that $U$ is in the basin of attraction for 0 . (We will view $U$ as a "fattened up" $W$.) Now, $\mathcal{A}-(\mathcal{A} \cap U)$ consists of three pairwise disjoint closed sets $R_{1}, R_{2}$, and $R_{3}$ each of which contains a pole (see Figure 6) with $R_{i} \subset P_{i}$ for $i=1,2,3$. Further, the $R_{i}$ can be chosen such that $G_{r}\left(R_{i}\right)$ covers $R_{1}, R_{2}$, and $R_{3}$ for any $i=1,2,3$. This arises from $P_{i} \subset G_{r}\left(P_{j}\right)$ for all $i, j=1,2,3$ and the fact that the portions of the $P_{i}$ where the map $G_{r}$ is not one-to-one occur on the boundary of $P_{i}$, which is not included in $R_{i}$. Hence, $J\left(G_{r}\right)$ consists of all points in $R_{1} \cup R_{2} \cup R_{3}$ that remain in $R_{1} \cup R_{2} \cup R_{3}$ for all iterations.

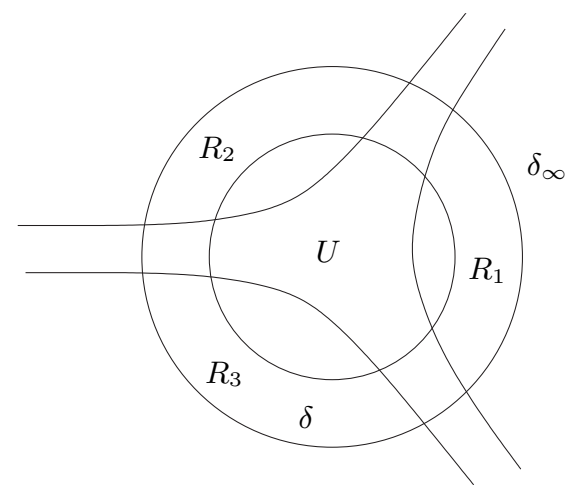

Fig. 6. The regions $R_{1}, R_{2}$, and $R_{3}$, the set $U$, and the curves $\delta$ and $\delta_{\infty}$

Theorem 2.2. For $r<r_{0}, J\left(G_{r}\right)$ is a Cantor set and $G_{r} \mid J\left(G_{r}\right)$ is conjugate to the one-sided shift on three symbols.

Proof. We need to show that each sequence corresponds to a point in the Julia set and that no sequence corresponds to multiple points. Since $G_{r}$ maps each $R_{i}$ over all of the $R_{i}$ 's we know that each sequence will correspond to at least one point in the Julia set.

Since the critical points are bounded away from the Julia set we know that $G_{r}$ is dynamically hyperbolic on its Julia set. This expansion guarantees that no sequence can correspond to multiple points. 
2.4. Structure of $J\left(G_{r}\right)$ for $r$ large. For $r<r_{0}$ there is a complexconjugate two-cycle with negative real part. This two-cycle is repelling for all $r<r_{0}$. However, our map undergoes a saddle-node bifurcation as $r$ passes through $r_{0}$ (see Figure 4) during which the repelling complex conjugate two-cycle coalesces into an indifferent fixed point on the negative real axis and then splits into two real fixed points on the negative real axis. We will denote these by $p$ and $q$, where $|q|<|p|$. For all $r>r_{0}, q$ is repelling and $p$ is attracting (superattracting if $r=3^{1 / 2} 2^{-1 / 6}$ ). There is also a repelling fixed point on the positive real axis which we will denote by $m$ (see Figure 7 ).

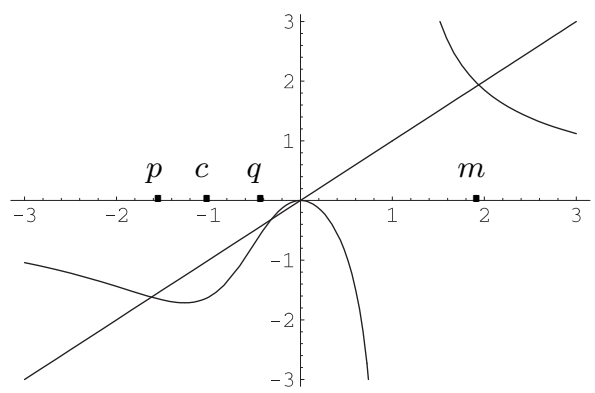

Fig. 7. The graph of $G_{r}$ for $r>r_{0}$

Since $G_{r}(\omega z)=\omega^{2} G_{r}(z)$ we know that there are actually three simultaneous bifurcations that occur as $r$ passes through $r_{0}$ : one on each of $\mathbb{R}^{-}, \omega \mathbb{R}^{-}$, and $\omega^{2} \mathbb{R}^{-}$. In the case of two generating circles, the bifurcation occurs when the radius of the generating circles is 1 ; in other words, when they are tangent. However, with three generating circles we should note that this bifurcation occurs when $r=r_{0}=3^{1 / 2} 2^{-1 / 3}$. The three generating circles become mutually tangent at $r=3^{1 / 2} 2^{-1}$. Hence the generating circles are actually overlapping when the bifurcation occurs (see Figure 8).
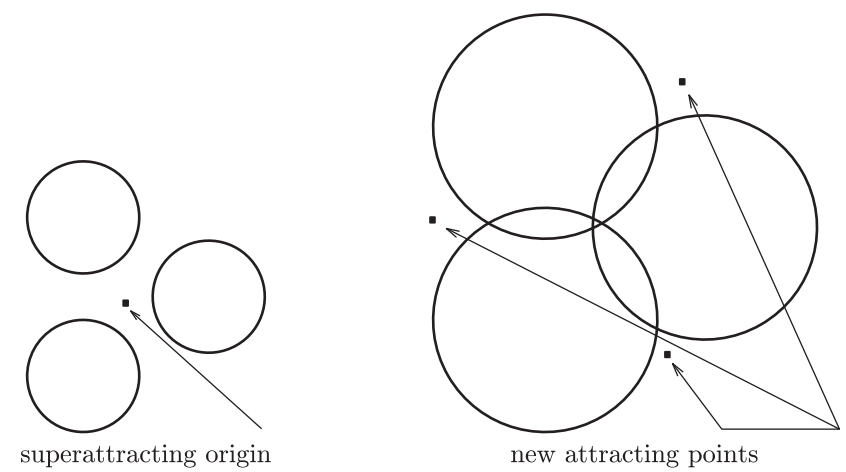

Fig. 8. The topological relation of the three generating circles pre- and post-bifurcation 
Note that the existence of an attracting fixed point on the negative real axis, namely $p$, implies the existence of an attracting two-cycle on the symmetric axes, namely the cycle consisting of the points $\omega p$ and $\omega^{2} p$. Let $A_{p}$ denote the immediate basin of attraction for $p$ and let $\mathcal{O}$ denote the immediate basin of attraction for 0 . Therefore, $\omega A_{p}$ and $\omega^{2} A_{p}$ form the immediate basin of attraction for the two-cycle formed by $\omega p$ and $\omega^{2} p$.

Theorem 2.3. The Fatou set is the union of $\mathcal{O}, A_{p}, \omega A_{p}, \omega^{2} A_{p}$ and all of their pre-images.

Proof. Since $p$ is an attracting fixed point there must be a critical point in $A_{p}$. Since $\mathbb{R}_{\infty}$ is forward and backward invariant this critical point must be either $c$ or 0 . Since 0 is fixed, we know $c \in A_{p}$. Hence, by the symmetries of $G_{r}$, we know that $\omega c$ and $\omega^{2} c$ are in $\omega A_{p} \cup \omega^{2} A_{p}$. Therefore we have all of the critical points for $G_{r}$ accounted for and there can be no other attracting cycles. This implies that all components of the Fatou set eventually iterate to one of $\mathcal{O}, A_{p}, \omega A_{p}$ or $\omega^{2} A_{p}$. This yields the desired result.

Since all of the critical points for $G_{r}$ are in basins of attraction we know $G_{r}$ is dynamically hyperbolic and that the immediate basins of attraction are simply connected. Therefore, all of their pre-images are simply connected, implying that every component of the Fatou set is simply connected. Theorem 5.1.6 in [1] then implies that the Julia set of $G_{r}(z)$ for $r>r_{0}$ is connected. It is known that if the Julia set of a dynamically hyperbolic map is connected then it is locally connected (see Theorem 19.2 in [3]). Therefore, $J\left(G_{r}\right)$ is locally connected as well as connected. The Julia sets for two of these maps are shown in Figure 9.
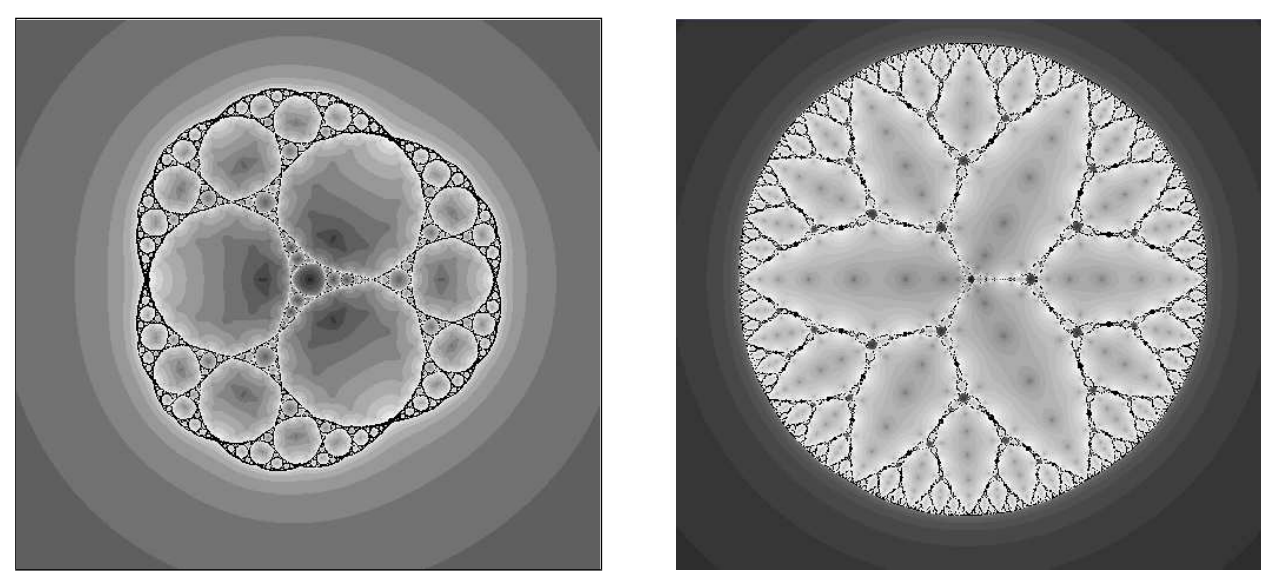

Fig. 9. The Julia sets for $G_{r}$ with $r=1.5$ and $r=2$ 
Since $G_{r}(\bar{z})=\overline{G_{r}(z)}$ we know that $\bar{A}_{p}=A_{p}, \overline{\mathcal{O}}=\mathcal{O}, \overline{\omega A_{p}}=\omega^{2} A_{p}$, and $\overline{\omega^{2} A_{p}}=\omega A_{p}$. Note $A_{p}$ cannot meet either of the symmetry axes, $\omega \mathbb{R}$ and $\omega^{2} \mathbb{R}$, because all points of $A_{p}$ are attracted to $p \in \mathbb{R}^{-}$while the union of the symmetry axes is forward invariant.

We will now show that the boundaries of $A_{p}$ and $\mathcal{O}$, denoted $\partial A_{p}$ and $\partial \mathcal{O}$, respectively, are simple closed curves.

TheORem 2.4. The boundary of $A_{p}$ is a simple closed curve.

Proof. Since $A_{p}$ is simply connected we know by Theorem 4.4.4 in [4] that the boundary $\partial A_{p}$ is a closed curve. Further, since our Julia set is locally connected we know that $\partial A_{p}$ is locally connected and Carathéodory theory tells us that $\phi$, the inverse of the Riemann map mapping $A_{p}$ to the unit disk $\mathbb{D}$, extends continuously to $\bar{\phi}: \overline{\mathbb{D}} \rightarrow \bar{A}_{p}$. We will call the images in $A_{p}$ of straight rays from 0 to $\partial \mathbb{D}$ internal rays. Since $\phi$ extends continuously to $\bar{\phi}$ we know that all of these rays land at a point on $\partial A_{p}$. To show that $\partial A_{p}$ is a simple closed curve we need only show that no two internal rays land at the same point in $\partial A_{p}$. Let us assume that two rays land at a point $w \in \partial A_{p}$. Now, let $\gamma$ be the Jordan curve consisting of these two rays along with the landing point $w$ and let $\Gamma$ denote the bounded complement of the curve $\gamma$ (see Figure 10). There must be other points of $\partial A$ in $\Gamma$, else we would have an entire sector of rays all landing at $w$, which is not possible.

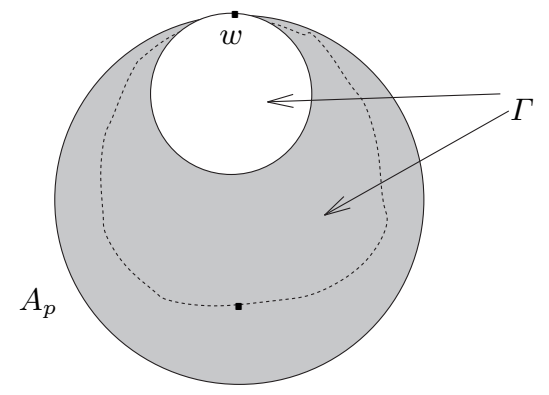

Fig. 10. The region $\Gamma$

Since $\Gamma$ is bounded by $\gamma$ we know by the maximum modulus theorem that $G_{r}(\Gamma)$ is bounded by $G_{r}(\gamma)$. (Note that $G_{r}(\gamma)$ is a simple closed curve.) Because $\gamma$ lies inside $A_{p}$, the basin of attraction for $p$ (with the exception of the point $w$ which lies on the boundary of this basin), we know that $G_{r}(\gamma-\{w\})$ lies inside $A_{p}$. Therefore, the boundary of $G_{r}(\Gamma)$ (with the exception of one point) lies inside $A_{p}$. Hence, $G_{r}(\Gamma)$ is either mapped to the unbounded complement of $G_{r}(\gamma)$ or to the bounded complement. It is known that any neighborhood of the Julia set for a rational map of degree $d \geq 2$ is eventually mapped by iterates of the map onto the entire 
Riemann sphere minus at most two points (see Theorems 4.1.2 and 4.2.5 in [1]). Since $\Gamma \cap J\left(G_{r}\right) \neq \emptyset$ it cannot be the case that $G_{r}(\Gamma)$ is mapped to the bounded complement of $G_{r}(\gamma)$. However, if $G_{r}(\Gamma)$ is mapped to the unbounded complement then $\Gamma$ must contain a pole. The poles lie on $\mathbb{R}^{+}$ and on the symmetric rays $t e^{2 \pi / 3}$ and $t e^{4 \pi / 3}$ with $t>0$. Since $p \in \mathbb{R}^{-}$we know that $A_{p}$ does not intersect $\mathbb{R}^{+}$or the symmetric rays. Hence, the pole must lie on the boundary of $A_{p}$, which is not possible. Note that if a pole did lie on the boundary of $A_{p}$ we could connect $p$ to the pole with a simple curve $\mu$ such that $\mu$ lies entirely within $A_{p}$. The image of $\mu$ would then be a simple curve connecting $\infty$ to $p$ contained entirely within $A_{p}$. This is not possible because there exist neighborhoods of $\infty$ consisting entirely of points in the basin of attraction for 0 . This contradiction establishes the result.

The same proof, with minor modifications, allows us to show that $\partial \mathcal{O}$ is also a simple closed curve. Symmetry allows us to extend this result to $\omega A_{p}$ and $\omega^{2} A_{p}$. Since all of our critical points tend to attracting cycles, we can generalize this result to all pre-images of attracting basins and hence to any component of the Fatou set. We have therefore shown:

TheOREM 2.5. If $C$ is the boundary of a Fatou component then $C$ is a simple closed curve.

There are also restrictions placed on the number of intersection points occurring between boundaries of pairwise disjoint complementary regions of the Julia set. Before continuing we will need the following lemma:

Lemma 2.6. The only point that remains in $\mathbb{R}^{+}$for all iterations is the repelling fixed point $m \in \mathbb{R}^{+}$.

Proof. It is easy to show that $G_{r}$ maps the interval $(0,1)$ onto $\mathbb{R}^{-}$. The pre-image of $\mathbb{R}^{+}$under $G_{r}(x)$ is the interval $(1, \infty)$. Further, $(1, \infty)$ is mapped one-to-one over $\mathbb{R}^{+}$. Let $d$ be the unique pre-image of 1 in $(1, \infty)$. Since the interval $(0,1)$ is mapped onto $\mathbb{R}^{-}$and the interval $(1, m)$ is mapped over $(m, \infty)$ (with $m>1$ ) we know that the point $d$ will always be in the interval $(m, \infty)$. The interval $(d, \infty)$ gets mapped over $(0,1)$ and hence is mapped to $\mathbb{R}^{-}$under the second iteration. Therefore, all points in $(0,1) \cup(d, \infty)$ are mapped to $\mathbb{R}^{-}$within two iterations of $G_{r}$. Finally, we look at the interval $(1, d)$. Since $m \in(1, d)$ we will look at the two intervals $(1, m)$ and $(m, d)$. (We know that $m$ remains in $\mathbb{R}^{+}$for all iterations and hence we only need to show that $(1, m) \cup(m, d)$ is mapped into $\mathbb{R}^{-}$.) Switching to the second iterate $G_{r}^{2}$, it becomes a simple matter to show that $(1, m)$ is mapped (by $G_{r}^{2}$ ) onto $(0, m)$ and $G_{r}^{2}(x)<x$ for all $x \in(1, m)$. Hence, all points in $(1, m)$ eventually iterate to $(0,1)$ and hence to $\mathbb{R}^{-}$. The interval $(m, d)$ is mapped (by $\left.G_{r}^{2}\right)$ onto $(m, \infty)$ and $G_{r}^{2}(x)>x$ for all $x \in(m, d)$. 
Hence, all of the points in $(m, d)$ eventually end up in $(d, \infty)$ and are then mapped into $\mathbb{R}^{-}$. Therefore all of the points in $\mathbb{R}^{+}-\{m\}$ are eventually mapped into $\mathbb{R}^{-}$.

We are now able to prove:

Theorem 2.7. If $\partial\left(\omega^{i} A_{p}\right)$ meets $\partial\left(\omega^{j} A_{p}\right)$ with $i, j=0,1,2$ and $i \neq j$, then it does so at exactly one point.

Proof. Note that $A_{p}$ is trapped in the region $S=\{z: \operatorname{Re}(z)<0,2 \pi / 3<$ $\operatorname{Arg}(z)<4 \pi / 3\}$. To see this note that $A_{p}$ cannot meet either of the symmetric axes since the symmetric axes are forward invariant and hence points on these axes cannot lie in the basin of attraction of a real non-zero fixed point. An analogous statement is true for the symmetric basins $\omega A_{p}$ and $\omega^{2} A_{p}$. We know that $\omega A_{p}$ must be trapped in $\omega S$ and $\omega^{2} A_{p}$ must be trapped in $\omega^{2} S$. Without loss of generality we will show that if $\partial\left(\omega A_{p}\right)$ meets $\partial\left(\omega^{2} A_{p}\right)$ then it does so at exactly one point. Since $\omega A_{p}$ is trapped in $\omega S$ and $\omega^{2} A_{p}$ is trapped in $\omega^{2} S$ we know that $\partial\left(\omega A_{p}\right) \cap \partial\left(\omega^{2} A_{p}\right)$ must lie in $\partial(\omega S) \cap \partial\left(\omega^{2} S\right)=\mathbb{R}^{+}$. Since $\omega A_{p}$ is mapped onto $\omega^{2} A_{p}$ and vice versa, it follows that $\partial\left(\omega A_{p}\right) \cap$ $\partial\left(\omega^{2} A_{p}\right) \subset \mathbb{R}^{+}$is mapped into $\mathbb{R}^{+}$. Hence, the set $\partial\left(\omega A_{p}\right) \cap \partial\left(\omega^{2} A_{p}\right)$ remains in $\mathbb{R}^{+}$for all iterations.

By Lemma 2.7 we know that the only point in $\mathbb{R}^{+}$that remains in $\mathbb{R}^{+}$ for all iterations is $m$. Hence, $\partial\left(\omega A_{p}\right) \cap \partial\left(\omega^{2} A_{p}\right)$ is either empty or contains only the positive repelling fixed point, $m$. Symmetry yields the result.

Let $S^{1}=\{z:|z|=1\}$ and $h(z)=z^{k}$. We will now make use of the following theorem which appears as Theorem 4.4.13 in [4]:

TheOREM 2.8. Assume that the Fatou set of a hyperbolic rational function $f$ contains a simply connected invariant component $D$ on which the local degree of $f$ is $k$. Then there is a continuous map $\phi$ from $S^{1}$ onto $\partial D$ such that

$$
\phi(h(z))=f(\phi(z)) .
$$

Moreover, $h$ is injective on $\phi^{-1}(\zeta)$ for all $\zeta \in \partial D$.

Using this theorem we will show:

Theorem 2.9. $\partial\left(\omega^{i} A_{p}\right) \cap \partial\left(\omega^{j} A_{p}\right) \neq \emptyset$ for $i \neq j$ and $i, j=0,1,2$.

Proof. $G_{r}$ is a hyperbolic rational map that maps $A_{p}$ onto $A_{p}$ in two-toone fashion. Hence, by Theorem 2.8, there exists a continuous map $\phi$ such that $\phi: S^{1} \rightarrow \partial A_{p}$ and

$$
\phi\left(z^{2}\right)=G_{r}(\phi(z)) .
$$

Therefore, $\phi\left(e^{4 \pi i / 3}\right)$ and $\phi\left(e^{2 \pi i / 3}\right)$ form a two-cycle on $\partial A_{p}$.

Since $G_{r}$ is of degree 3 we know that there are at most three distinct two-cycles. Since $q$ is a repelling fixed point on the boundary of $A_{p}$ we know 
that $\omega q$ and $\omega^{2} q$ form a repelling two-cycle with $\omega q \in \omega\left(\partial A_{p}\right)=\partial\left(\omega A_{p}\right)$ and $\omega^{2} q \in \omega^{2}\left(\partial A_{p}\right)=\partial\left(\omega^{2} A_{p}\right)$. Hence, the attracting two-cycle $\left\{\omega p, \omega^{2} p\right\}$ and the repelling two-cycle $\left\{\omega q, \omega^{2} q\right\}$ both have positive real components. However, $\partial A_{p}$ is entirely contained in the left half-plane $\{z: \operatorname{Re}(z)<0\}$. Therefore the two-cycle on $\partial A_{p}$ must be distinct from the previous twocycles, giving us our three two-cycles.

Recall that we denote the repelling fixed point on the positive real axis by $m$. Therefore, $\omega m$ and $\omega^{2} m$ form a two-cycle with negative real component. Since we have all three of our two-cycles accounted for, it must be that $\left\{\omega m, \omega^{2} m\right\}$ corresponds to the two-cycle in $\partial A_{p}$. We have $\omega m \in \partial A_{p}$ and therefore, by the symmetries, we know that $\omega^{2} m \in \omega\left(\partial A_{p}\right)=\partial\left(\omega A_{p}\right)$. However, $\omega^{2} m$ is also in $\partial A_{p}$. Hence, $\omega^{2} m \in \partial A_{p} \cap \omega\left(\partial A_{p}\right)=\partial A_{p} \cap \partial\left(\omega A_{p}\right)$. This implies that $\omega m \in \partial A_{p} \cap \omega^{2}\left(\partial A_{p}\right)=\partial A_{p} \cap \partial\left(\omega^{2} A_{p}\right)$ and, finally, that $m \in \omega\left(\partial A_{p}\right) \cap \omega^{2}\left(\partial A_{p}\right)=\partial\left(\omega A_{p}\right) \cap \partial\left(\omega^{2} A_{p}\right)$. Hence, $\partial\left(\omega^{i} A_{p}\right) \cap \partial\left(\omega^{j} A_{p}\right) \neq \emptyset$ for $i \neq j$.

Combining Theorems 2.7 and 2.9 we obtain:

Corollary 2.10. $\partial\left(\omega^{i} A_{p}\right)$ meets $\partial\left(\omega^{j} A_{p}\right)$ at exactly one point for $i, j=$ $0,1,2$ and $i \neq j$.

We will now show that a similar result holds for $\mathcal{O}$. Recall that $q$ is the repelling fixed point on $\mathbb{R}^{-}$.

Lemma 2.11. For all $r>r_{0}$ we have $q \in \partial A_{p} \cap \partial \mathcal{O}$.

Proof. The fixed point $q$ is given by

$$
q=q_{r}=\frac{(-3 i+\sqrt{3}) r^{2}+(-2)^{1 / 3} 3^{1 / 6}\left(-9+\sqrt{81-12 r^{6}}\right)^{2 / 3}}{2^{2 / 3} 3^{5 / 6}\left(-9+\sqrt{81-12 r^{6}}\right)^{1 / 3}},
$$

while the fixed point $p$ is given by

$$
p=p_{r}=-\frac{2 \cdot 3^{1 / 3} r^{2}+2^{1 / 3}\left(-9+\sqrt{81-12 r^{6}}\right)^{2 / 3}}{6^{2 / 3}\left(-9+\sqrt{81-12 r^{6}}\right)^{2 / 3}} .
$$

A simple calculation shows that $p$ and $q$ are complex for $r<r_{0}$. When $r=r_{0}$ we have $p=q<0$, and when $r>r_{0}$ we have $p<q<0$.

When $q<x<0$ we have $x^{3}-r^{2} x-1<0$. This implies that $x\left(x^{3}-r^{2} x-1\right)$ $>0$ since $x<0$. Hence, $r^{2} x^{2}<x\left(x^{3}-1\right)$, implying $\left|r^{2} x^{2}\right|<|x|\left|x^{3}-1\right|$, since $x$ and $x^{3}-1$ are negative. This implies that

$$
\left|\frac{r^{2} x^{2}}{x^{3}-1}\right|<|x| \text {. }
$$

Hence, for all $x \in(q, 0)$ and $r>r_{0}$ we have $\left|G_{r}(x)\right|<|x|$. This implies that all points in $(q, 0)$ are in $\mathcal{O}$. Now we need to show that the interval $(p, q)$ is in $A_{p}$. Recall that the point $p$ is superattracting if $r=3^{1 / 2} 2^{-1 / 6}$. For $3^{1 / 2} 2^{-1 / 3}=r_{0}<r<3^{1 / 2} 2^{-1 / 6}$ the critical value $c$ is less than $p$. In 
this case for all points $x$ in $(p, q)$ we have $p<G_{r}(x)<x$, implying that $(p, q) \in A_{p}$.

If $r \geq 3^{1 / 2} 2^{-1 / 6}$ then $c>p$. For these $r$-values $G_{r}(x)$ is continuous with no critical points on the interval $(p, c)$. Hence, the interval $(p, c)$ is mapped to the interval $\left(G_{r}(c), G_{r}(p)\right)=\left(G_{r}(c), p\right)$. Continuing, the image of the interval $(p, c)$ under $G_{r}^{n}(x)$ is the interval $\left(G_{r}^{n}(c), p\right)$ (if $n$ is odd) or $\left(p, G_{r}^{n}(c)\right)$ (if $n$ is even). Since $c$ is in the immediate basin of attraction for $p$ we know that as $n \rightarrow \infty$ we have $G_{r}^{n}(c) \rightarrow p$. Hence, $G_{r}^{n}(x) \rightarrow p$ as $n \rightarrow \infty$ for all $x \in(p, c)$, implying that $(p, c) \subset A_{p}$. Since $G_{r}^{\prime}(q)>1$ and there are no fixed points or poles in $(c, q)$ and $c$ is the local minimum point we know that $G_{r}(c)<G_{r}(x)<x$ for all $x \in(c, q)$. Hence, all of these $x$-values will eventually iterate into the interval $\left(G_{r}(c), c\right) \subset A_{p}$, so $(c, q) \subset A_{p}$. Since $c$ is in $A_{p}$ this implies that $(p, q) \subset A_{p}$.

Therefore, $(p, q) \subset A_{p}$ and $(q, 0) \subset \mathcal{O}$, and consequently $q \in \partial A_{p} \cap \partial \mathcal{O}$.

Theorem 2.12. $\partial \mathcal{O}$ meets $\partial\left(\omega^{i} A_{p}\right)$ at exactly one point for $i=0,1,2$.

Proof. From the lemma we know $q \in \partial A_{p} \cap \partial \mathcal{O}$. We claim that $\partial A_{p} \cap \partial \mathcal{O}=\{q\}$. Assume that there exists another point, $\alpha \in \partial A_{p} \cap \partial \mathcal{O}$. Since $\bar{A}_{p}=A_{p}$ and $\overline{\mathcal{O}}=\mathcal{O}$ we know that $\bar{\alpha} \in \partial A_{p} \cap \partial \mathcal{O}$ as well. Let $\gamma_{A}$ represent the portion of $\partial A_{p}$ between $\alpha$ and $\bar{\alpha}$ containing $q$. Now let $(\alpha, q)_{A}$ be the portion of $\gamma_{A}$ connecting $\alpha$ to $q$ and $(q, \bar{\alpha})_{A}$ the portion connecting $q$ to $\bar{\alpha}$. In a similar fashion we can define $\gamma_{\mathcal{O}}$ and its subsets $(\alpha, q)_{\mathcal{O}}$ and $(q, \bar{\alpha})_{\mathcal{O}}$. Denote by $\Gamma_{\text {upper }}$ the bounded complement of the closed curve $\{\alpha\} \cup\{q\} \cup(\alpha, q)_{A} \cup(\alpha, q)_{\mathcal{O}}$ and $\Gamma_{\text {lower }}$ the bounded complement of the closed curve $\{q\} \cup\{\bar{\alpha}\} \cup(q, \bar{\alpha})_{A} \cup(q, \bar{\alpha})_{\mathcal{O}}$. Let $\Gamma=\Gamma_{\text {upper }} \cup \Gamma_{\text {lower }}$ (see Figure 11). Note that $\Gamma \neq \emptyset$. If $\Gamma$ were empty this would imply that $\partial A_{p}$ and $\partial \mathcal{O}$ meet at an arc contained in the Julia set. We can then define $N$, a neighborhood of $q$, such that $N$ is contained in $A_{p} \cup \mathcal{O} \cup\left(\partial A_{p} \cap \partial \mathcal{O}\right)$. Since $N$ is a neighborhood of the Julia set we know that the iterates of $N$ must eventually cover the entire Riemann sphere minus at most two points. This is not possible since the intersection of $N$ and the Fatou set is contained in the forward invariant set $A_{p} \cup \mathcal{O}$. Hence, $N$ would never iterate to cover points contained in $\omega A_{p}$ or $\omega^{2} A_{p}$. This contradiction establishes that $\Gamma$ must be non-empty.

Since $\partial A_{p}$ and $\partial \mathcal{O}$ are forward invariant, $G_{r}\left(\partial \Gamma_{\text {upper }}\right) \subset \partial A_{p} \cup \partial \mathcal{O}$ and the same is true for $\partial \Gamma_{\text {lower }}$. Hence, $G_{r}\left(\Gamma_{\text {upper }}\right)$ and $G_{r}\left(\Gamma_{\text {lower }}\right)$ are either contained within $\overline{A_{p} \cup \Gamma \cup \mathcal{O}}$ or are unbounded.

The first is impossible for it would imply that $G_{r}^{n}\left(\Gamma_{\text {lower }}\right) \subset \overline{A_{p} \cup \Gamma \cup \mathcal{O}}$ for all $n>0$. Hence, we can take a neighborhood $N$ of $\partial \Gamma_{\text {lower }}$ that contains only points from $\overline{A_{p} \cup \Gamma \cup \mathcal{O}}$. This neighborhood is contained in $\overline{A_{p} \cup \Gamma \cup \mathcal{O}}$ for all iterations. This is a contradiction because $N$ is a neighborhood containing points in the Julia set of $G_{r}$ (namely $\partial \Gamma_{\text {lower }} \cap N$ ) and therefore its 


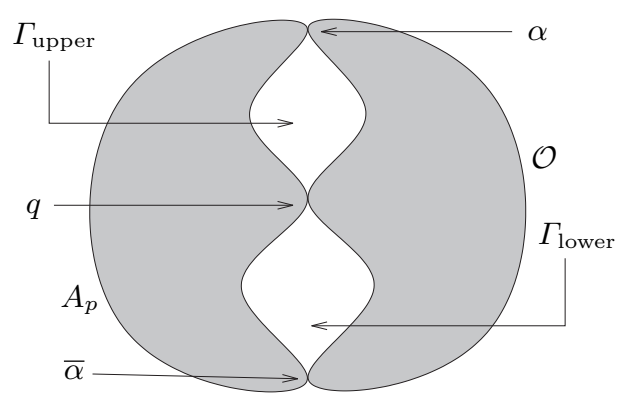

Fig. 11. $\partial A_{p}$ meets $\partial \mathcal{O}$ at three points

iterates must eventually cover the entire Riemann sphere minus at most two points. The same argument holds for $\Gamma_{\text {upper }}$.

Hence, it must be the case that $G_{r}\left(\Gamma_{\text {upper }}\right)$ and $G_{r}\left(\Gamma_{\text {lower }}\right)$ are unbounded. Therefore, both $\Gamma_{\text {upper }}$ and $\Gamma_{\text {lower }}$ contain poles and both of these regions are incident to $A_{p}$ and $\mathcal{O}$ only. Hence, for $i=0,1,2$ we have two sets, $\omega^{i}\left(\Gamma_{\text {upper }}\right)$ and $\omega^{i}\left(\Gamma_{\text {lower }}\right)$, that are distinct and contain poles. This implies that our degree 3 map has at least six poles. This contradiction establishes the result.

2.5. Dynamics of $G_{r}$ on $J\left(G_{r}\right)$. We shall now describe the dynamics of $G_{r}$ on $J\left(G_{r}\right)$ via symbolic dynamics. For $r<r_{0}$ we know that $G_{r} \mid J\left(G_{r}\right)$ is conjugate to the shift map on three symbols. Now let us consider $r>r_{0}$. Consider the closed curve $\partial \mathcal{O}$ encircling zero. Its pre-image consists of two closed curves: $\partial \mathcal{O}$ itself and a closed curve surrounding $\infty$ that we will denote by $\partial \mathcal{O}_{\infty}^{-1}$. Note that $\partial \mathcal{O}$ is mapped in two-to-one fashion over itself making two counterclockwise twists around the origin, while $\partial \mathcal{O}_{\infty}^{-1}$ is mapped in oneto-one fashion over $\partial \mathcal{O}$ making one clockwise twist around the origin. All points outside $\partial \mathcal{O}_{\infty}^{-1}$ and inside $\partial \mathcal{O}$ are attracted to the origin. Therefore, all of our interesting dynamics occurs in the annular region between $\partial \mathcal{O}$ and $\partial \mathcal{O}_{\infty}^{-1}$. We will denote this region by $R$.

Theorem 2.13. $\partial\left(\omega^{i} A_{p}\right)$ meets $\partial\left(\mathcal{O}_{\infty}^{-1}\right)$ at exactly one point for $i, j=$ $0,1,2$.

Proof. Let the pre-image of $q$ lying on the negative real axis be denoted $q^{-1}$. Note that $q^{-1}<q$ and the interval $\left(-\infty, q^{-1}\right)$ is mapped over the interval $(q, 0)$. However, $(q, 0) \subset \mathcal{O}$ (see proof of Lemma 2.11). Hence, $q^{-1} \in$ $\partial \mathcal{O}_{\infty}^{-1}$. Further, the interval $\left(q^{-1}, p\right)$ is mapped onto $(p, q)$ and $(p, q) \subset A_{p}$ (see proof of Lemma 2.11). Therefore, $q^{-1} \in \partial A_{p}$. Hence, $\partial A_{p}$ meets $\partial \mathcal{O}_{\infty}^{-1}$ at exactly one point along the negative real axis. By symmetry, similar results hold for $\partial\left(\omega A_{p}\right)$ and $\partial\left(\omega^{2} A_{p}\right)$. 
Let us consider the region obtained from $R$ by removing the open sets $A_{p}, \omega A_{p}$ and $\omega^{2} A_{p}$. We will cut this region into three parts with the rays $\arg z=\pi / 3, \arg z=\pi$ and $\arg z=5 \pi / 3$. We will call these regions $R_{1}$, $R_{2}$, and $R_{3}$, with $R_{1}$ between $\arg z=5 \pi / 3$ and $\arg z=\pi / 3, R_{2}$ between $\arg z=\pi / 3$ and $\arg z=\pi$, and $R_{3}$ between $\arg z=\pi$ and $\arg z=5 \pi / 3$ (see Figure 12).

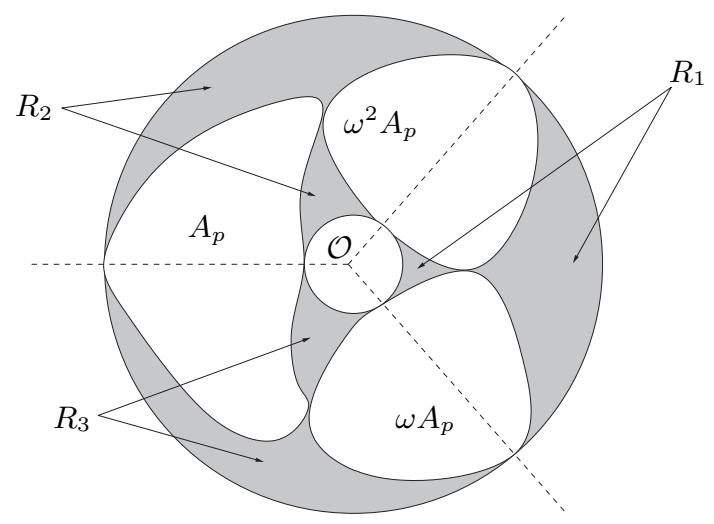

Fig. 12. The regions $R_{1}, R_{2}, R_{3}$

Note that it follows from Corollary 2.10 and Theorems 2.12 and 2.13 that only six points lie in multiple regions. The repelling fixed point $q$ and its pre-image $q^{-1}$ on $\mathbb{R}^{-}$are the only points in $R_{2} \cap R_{3}$. We have exactly two points in $R_{1} \cap R_{2}$, namely $\omega^{2} q$ and $\omega^{2} q^{-1}$, and exactly two points in $R_{3} \cap R_{1}$, namely $\omega q$ and $\omega q^{-1}$. Each $R_{i}(i=1,2,3)$ is mapped in one-to-one fashion over $R_{1}, R_{2}$ and $R_{3}$ by $G_{r}$. The argument used to show this is the same as the argument used to show the similar result in Section 2.3, except that $D$ is replaced by $\mathcal{O}$.

We are now in a position to describe the dynamics of $G_{r}$ on $J\left(G_{r}\right)$ using symbolic dynamics on the regions $R_{1}, R_{2}$, and $R_{3}$. To each point $z \in J\left(G_{r}\right)$ we can assign a sequence $s(z)=s_{1} s_{2} s_{3} \ldots$ using the rule $G_{r}^{i}(z) \in R_{s_{i}}$. Since six points lie in multiple regions those points (and all of their pre-images) will be associated with multiple sequences. Hence, we will be able to show that $G_{r} \mid J\left(G_{r}\right)$ is conjugate to a quotient of a one-sided shift on three symbols.

THEOREM 2.14. There is a semiconjugacy between $G_{r} \mid J\left(G_{r}\right)$ and a quotient of the one-sided shift map on three symbols. The semiconjugacy is given by associating to each point $z \in J\left(G_{r}\right)$ a sequence $\left\{s_{0}, s_{1}, \ldots\right\}$ where $s_{n}=1,2,3$ and is given by $G_{r}^{n}(z) \in R_{s_{n}}$.

Proof. We need to show that each sequence corresponds to at least one point in the Julia set and that no sequence corresponds to multiple points. 
Since each region $R_{i}$ is mapped over all three regions we are guaranteed that each sequence will correspond to at least one point in the Julia set.

The Julia set is contained in $\bigcup R_{i}$ but $\bigcup R_{i}$ contains no critical points. Hence we know that the critical points are disjoint from the Julia set, implying that $G_{r}$ is dynamically hyperbolic on its Julia set. This expansion guarantees that no sequence can correspond to multiple points.

We only have a semiconjugacy and not a conjugacy due to the six points that lie in multiple regions. Any sequence ending with $\{111111 \ldots\}$ is equivalent to the same sequence ending with $\{232323 \ldots\}$ or $\{323232 \ldots\}$, and any sequence ending with $\{222222 \ldots\}$ is equivalent to the same sequence ending in $\{333333 \ldots\}$. The former of these identifications corresponds to the two-cycle $\left\{\omega q, \omega^{2} q\right\}$ and its pre-images, while the latter corresponds to the fixed point $q$ and its pre-images. If we exclude these cases our semiconjugacy becomes a conjugacy.

\section{References}

[1] A. F. Beardon, Iteration of Rational Functions, Springer, 1991.

[2] R. L. Devaney, An Introduction to Chaotic Dynamical Systems, Addison-Wesley, 1989.

[3] J. Milnor, Dynamics in One Complex Variable, Vieweg, 1999.

[4] S. Morosawa, Y. Nishimura, M. Taniuchi and T. Ueda, Holomorphic Dynamics, Cambridge Univ. Press, 2000.

Room 233 Stright Hall

Indiana University of Pennsylvania

Indiana, PA 15705, U.S.A.

E-mail: lookd@iup.edu

Received 18 March 2007;

in revised form 5 September 2007 